\title{
Spontaneous hepatic duct perforation in pregnancy: a case report and review of the literature
}

Published online: 13 October 2004

(C) Springer-Verlag Berlin / Heidelberg 2004

\begin{abstract}
Spontaneous perforation of the hepatic duct is a rare disease, and occurrence during pregnancy is even rarer. Treatment during pregnancy is crucial for both the mother and fetus. However, the treatment is a subject of controversy. We will discuss the case of an 18-year-old G1P1, 31 weeks pregnant, who showed acute onset of abdominal pain with peritoneal signs. She was taken to the operating room for exploratory laparotomy and was discovered to have a perforation of the common hepatic duct. Several factors thought to have caused spontaneous rupture of the bile tract were present: biliary calculi, infection, vessel thrombosis and pregnancy. The patient underwent a cholecystectomy, choledochotomy with removal of gallstones, repair of the perforation and placement of a T-tube. The postoperative course passed without problems for the mother and fetus. There were no complications, and the woman successfully carried her pregnancy to term. We conclude that the treatment of this unique and highly dangerous complication can be applied with an excellent outcome. The mechanism in this rare condition is discussed and reviewed.
\end{abstract}

Keywords Perforation of the hepatic duct - Pregnancy Cholecystectomy · Choledochotomy $\cdot$ T-tube

\section{Introduction}

Spontaneous rupture of the common hepatic duct is a rare condition $[1,2,3,4,5]$, and occurrence during pregnancy is even rarer [6]. The process of rupture is often obscure, and clinical experience is still limited, even more so when dealing with this condition in pregnancy. Commonly, the gallbladder is the site of non-traumatic perforation in

T. Sauper $\cdot$ M. Lanthaler $\cdot$ E. Weissenboeck $\cdot$ H. Nehoda $(\square)$ Department of General and Transplant Surgery,

University Hospital of Innsbruck,

Anichstrasse 35, 6020 Innsbruck, Austria

e-mail: hermann.nehoda@uibk.ac.at

Tel.: +43-512-5042592

Fax: +43-512-5042575 association with gallstone disease, but perforation of the common bile duct and the hepatic duct also occurs. Hepatic duct rupture is extremely rare and has been recorded on only 29 occasions in adults $[1,7,8]$. Early diagnosis and an aggressive approach to therapy are mandatory to alleviate sepsis and achieve decompression of the biliary duct system.

\section{Case report}

An 18-year-old G1P1, 32 weeks pregnant, was admitted to our unit with a 1-day history of continuous upper abdominal pain and fever $\left(38.5^{\circ} \mathrm{C}\right)$. The patient had neither a vaginal discharge nor bleeding, and fetal movements were normal. On examination, her pulse was $88 \mathrm{bpm}$ and her blood pressure was $120 / 75 \mathrm{mmHg}$. Abdominal examination revealed rebound tenderness in the upper abdomen. The uterus was 31-weeks size on palpation. Fetal heart sounds were normal. Bowel sounds were normal on auscultation. Rectal examination was also normal.

Sonography of the abdomen revealed cholelithiasis (between two and three calculi), signs of cholecystitis, a dilated common bile duct with a diameter of $0.5 \mathrm{~cm}$ and a perihepatic fluid collection (2$3 \mathrm{~mm}$ ). The uterus was normal with a living, 31-week-old fetus. Hemoglobin was $11 \mathrm{~g} / \mathrm{dl}$, and the white cell count was 15,700 cells/ $\mathrm{mm}^{3}$. The liver profile was elevated (GOT 27, GPT 35, yGT 94, alkaline phosphates 181), and total bilirubin, serum amylase and renal profile were within the normal limits. Preoperatively, the patient was examined three times within $36 \mathrm{~h}$ to exclude an acute abdomen and in order to ensure fetal lung maturity. The abdominal sonogram revealed little free fluid. Additionally, the symptoms the patient showed improved. Consequently, no grounds for earlier surgical intervention were seen as necessary. On day 3 , the patient's condition deteriorated suddenly, and surgical exploration was immediately performed. Laparotomy disclosed biliary peritonitis. There were $300 \mathrm{ml}$ of clear yellow bile in the supracolic compartment along with bile staining of the common hepatic duct (Fig. 1) together with several calculi originating from a 4-5-mm perforation at the confluence of the right and left hepatic duct (Fig. 2). The gallbladder showed signs of chronic cholecystitis. Cholecystectomy and exploration of the common duct were performed. The gallstones were removed. After removal of several common duct stones, it was decided temporarily to drain the common hepatic duct with a T-tube drain $(3.5 \mathrm{~mm})$, and wide drainage of the peritoneal cavity was performed. Antibiotic therapy of ampicillin and clavulanacid was initiated.

The T-tube drain was left in situ for 10 days, being clamped intermittently beginning on the 7 th postoperative day. T-tube 


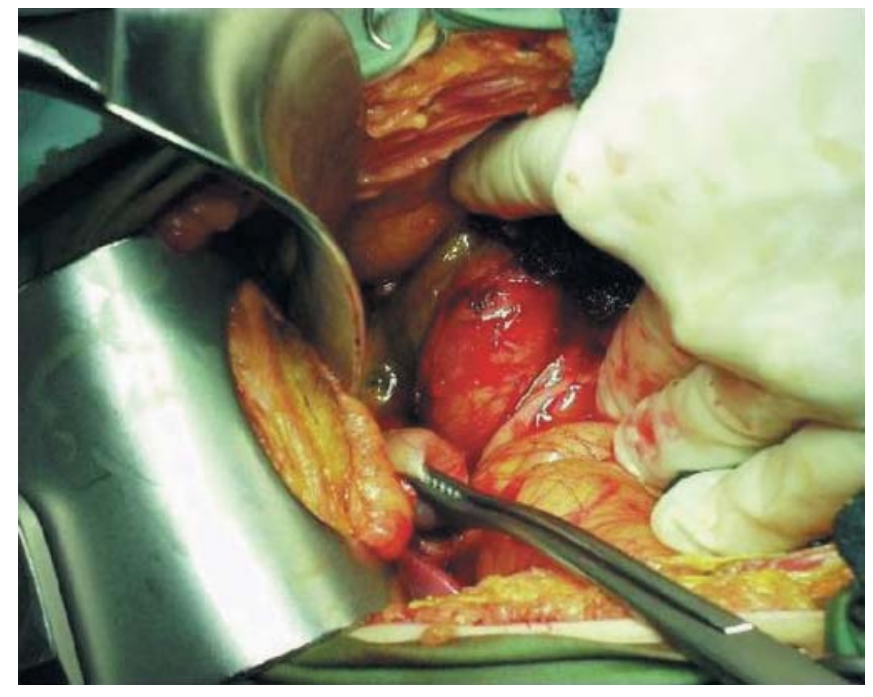

Fig. 1 Yellow bile in the supracolic compartment along with bile staining of the common hepatic duct

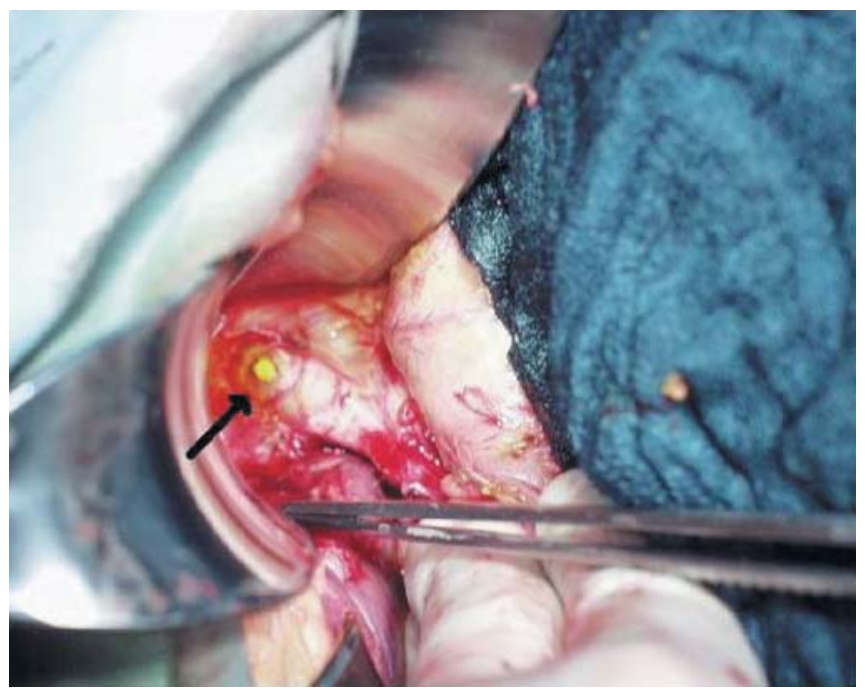

Fig. 2 Several calculi originating from a 4-5 $\mathrm{mm}$ perforation at the confluence of the right and left hepatic ducts

cholangiogram was not performed because of pregnancy. The postoperative course passed without problems, and the patient was discharged symptom free, with moderately elevated liver enzymes only. At follow-up 4 weeks later, the patient was still asymptomatic with normal liver function tests and no evidence of bile duct stricture on abdominal ultrasonography. The woman successfully carried her pregnancy to term. Six weeks after being discharged, she had a normal vaginal delivery. Repeat postoperative control visits included ultrasound examination, which revealed no signs of incisional hernia.

\section{Discussion}

The rarity of spontaneous perforation of the hepatic duct in the absence of trauma or previous biliary surgery is apparent by the few recorded reports. Only 29 cases have been reported in adults since 1882 [1, 7, 8]. Most patients were elderly, and the clinical presentation was nearly the same in all cases. Diagnosis was not made before taking the patient to the operating room for exploratory laparotomy. The sonogram of the abdomen revealed no signs of perforation. The duration of symptoms was prolonged. The patients displayed abdominal distension, jaundice and cholangitis. Alkaline phosphates levels were usually elevated.

The most common site of perforation is the common hepatic duct $[1,7,8]$. Twenty patients have been documented with this, including our patient. Perforation was reported to range from $2 \mathrm{~mm}$ to $1 \mathrm{~cm}$.

The putative causes of biliary duct perforation described in the surgical literature are stone erosion, stones, superimposed infection and cirrhosis. Choledochal calculi associated with infected bile as well as increased pressure in the proximal ductal system are the most common etiological factors in spontaneous perforation of biliary ducts [3]. One theory documented in the medical literature [1] is that obstruction of the biliary tract by gallstones results in raised intraductal pressure, leading to dilatation of the biliary tree, subsequent stasis and infection; this causes ascending cholangitis and thrombosis of intramural vessels, leading to necrosis and perforation of the duct wall. The reason why perforation is so uncommonly associated with obstructive jaundice due to tumor [9] is also discussed. Authors suspect that the slow rise in intraductal pressure is less likely to cause rupture than a sudden increase, which is usually seen with stone disease. In our case, biliary calculi could be observed seeping from the leakage site. It has been stated that spontaneous bile duct perforation of either the hepatic or common bile duct is due to single or multiple factors. The pathogenesis of nontraumatic perforation of the biliary tract system is poorly understood, but the following processes are generally accepted [2]: (1) calculous perforation at the site of impaction or erosion without evidence of mechanical disproportion of the ductal system; (2) increased intracanalicular pressure due to obstruction by the tumor, impacted stone or spasm of the sphincter of Oddi; (3) intramural infection; (4) intramural infarction due to thrombosis of the mural vessels; (5) rupture of a diverticulum or a cyst of the biliary tract; (6) regurgitation of activated pancreatic juice through a common outlet of the common bile duct and the pancreatic duct; (7) traction from neighboring organs. However, there is little evidence to support any of these theories.

We report the case of a pregnant woman who showed an acute abdomen after spontaneous perforation of the common hepatic duct. The effect of pregnancy on the occurrence of gallstone disease has been documented [10, $11,12,13,14,15,16]$. Women are more likely to develop gallstones than men $[16,17]$. This sex-related difference in incidence is thought to be related to pregnancy. There are several reports that suggest that the lithogenicity of estrogen is related to the increase in content of cholesterol in bile. Other studies report that the emptying of the 
gallbladder becomes poor during pregnancy $[11,12,13$, 14]. Moreover, pregnant women gain weight, and it has been well known that obesity is a risk factor in the development of gallstones ever since Deaver first characterized patients at risk as "female, fair, fat, forty and fertile" [18]. In all, reviewing the literature $[11,12,13$, $14,15,16]$, pregnancy is a very important pathogenic factor favoring gallstone formation.

We believe that in the case of our patient, the elevated intraductal pressure because of stone impaction at the weakest point of the biliary duct system and the possible hemodynamic changes associated with the higher pressure in the vena cava occurring during pregnancy could be a probable cause of bile duct perforation. As pregnancy is a very important factor favoring gallstone formation, we would like to emphasize that every surgeon should be aware of the possibility of bile duct perforation if a woman displays acute abdomen during pregnancy.

Reviewing the literature, treatment of the perforations of the bile duct system were extremely varied, inluding: (1) fine suture closure, (2) T-tube decompression of the biliary tree and drainage of the right upper quadrant, (3) common duct exploration and the T-tube placement through the perforation itself, as we successfully did in our patient, including cholecystectomy, and (4) Roux-en$\mathrm{Y}$ ductal anastomosis if ductal disruption is severe.

Most authors advocate fine-suture closure with care to avoid narrowing the duct. Our experience seems to suggest that suture closure is not mandatory, since in two instances T-tube decompression was done successfully and narrowing of the duct did not occur.

Common sense and widely accepted principles of biliary tract surgery are certainly central to minimizing the mortality and morbidity rates in this setting. Adequate drainage of the right upper quadrant is important, as is parenteral antibiotic therapy with agents specific for bile flora. A T-tube should be left in place for several days or weeks. Premature removal may result in a bile leak. During pregnancy, preoperative and postoperative fetal monitoring and adequate management of anesthetic and tocolytic agents are absolutely necessary. In a similar situation, many colleagues recommend combining laparotomy with caesarean section as the appropriate management in order to provide maximum mother and child safety. As seen in our case, a separate procedure followed by vaginal delivery at term is possible and safe for both mother and child. It would appear that bile duct perforations can be treated successfully if the patient's condition is not compromised too much by an undue delay prior to the operation.

\section{References}

1. Paladugu R, Rau A, Schein M, Wise L (1998) Spontaneous perforation of the hepatic duct in adults. Dig Surg 15:417-420

2. Christensen AB, Baekgaard N, Blichert-Toft M (1977) Bile peritonitis due to spontaneous perforation of the common hepatic duct. Report of a case. Acta Chir Scand 143:495-497

3. Donald JW, Ozment ED Jr (1977) Spontaneous perforation of bile ducts. Am Surg 43:524-527

4. Kyzer S, Bayer I, Chaimoff C (1986) Spontaneous rupture of the common bile duct. Can J Surg 29:68-69

5. Nomura T, Nikkuni K, Kato H, et al (1926) Bile peritonitis due to a spontaneous perforation of the common bile duct: report of a case. Surg Today 26:822-824

6. Piotrowski JJ, Van Stiegmann G, Liechty RD (1990) Spontaneous bile duct rupture in pregnancy. HPB Surg 2:205-209

7. Nguyen WD, Daza E (2001) Spontaneous perforation of the right hepatic duct. Hepatogastroenterology 48:1028-1029

8. Wang CH, Lin RC, Mo LR, Yau MP, Tsai CC (1995) Spontaneous perforation of the left hepatic duct-a case report. Hepatogastroenterology 42:77-79

9. Hill NS, Colapinto ND (1981) Spontaneous perforation of the common bile duct. Br J Surg 68:661-662

10. Valdivieso V, Covarrubias C,Siegel F, Cruz F (1993) Pregnancy and cholelithiasis: pathogenesis and natural course of gallstones diagnosed in early puerperium. Hepatology 17:1-4

11. Gilat T, Konikoff F (2000) Pregnancy and the biliary tract. Can J Gastroenterol 14 [Suppl D]:55D-59D

12. Scott LD (1992) Gallstone disease and pancreatitis in pregnancy. Gastroenterol Clin North Am 21:803-815

13. Everson GT (1992) Gastrointestinal motility in pregnancy. Gastroenterol Clin North Am 21:751-776

14. Weiss GN, Weiss EB (1976) Hormonal therapy and cholelithiasis. Int Surg 61:472-474

15. Everson GT, McKinley C, Lawson M, Johnson M, Kern F Jr (1982) Gallbladder function in the human female: effect of the ovulatory cycle, pregnancy, and contraceptive steroids. Gastroenterology 82:711-719

16. Tsimoyiannis EC, Antoniou NC, Tsaboulas C, Papanikolaou N (1994) Cholelithiasis during pregnancy and lactation. Eur J Surg 160:627-631

17. Jorgensen T, Jensen KH (1992) Who has gallstones? Current epidemiologic studies. Nord Med 107:122-125

18. Deaver JB (1930) Sequelae of biliary tract infection. JAMA 95:1644-1650 